Portland State University

PDXScholar

\title{
Bioelectrical Impedance Analysis as a Laboratory Activity: At the Interface of Physics and the Body
}

\author{
Elliot Mylott \\ Portland State University \\ Ellynne Marie Kutschera \\ Portland State University \\ Ralf Widenhorn \\ Portland State University
}

Follow this and additional works at: https://pdxscholar.library.pdx.edu/phy_fac

Part of the Physics Commons, and the Science and Mathematics Education Commons Let us know how access to this document benefits you.

\section{Citation Details}

Mylott, E., Kutschera, E., \& Widenhorn, R. (2014). Bioelectrical impedance analysis as a laboratory activity: At the interface of physics and the body. American Journal Of Physics, 82(5), 521-528.

This Article is brought to you for free and open access. It has been accepted for inclusion in Physics Faculty Publications and Presentations by an authorized administrator of PDXScholar. Please contact us if we can make this document more accessible: pdxscholar@pdx.edu. 


\title{
Bioelectrical impedance analysis as a laboratory activity: At the interface of physics and the body
}

\author{
Elliot Mylott, ${ }^{\text {a) }}$ Ellynne Kutschera, and Ralf Widenhorn \\ Department of Physics, Portland State University, Portland, Oregon 97207
}

(Received 22 August 2013; accepted 7 February 2014)

\begin{abstract}
We present a novel laboratory activity on $R C$ circuits aimed at introductory physics students in life-science majors. The activity teaches principles of $R C$ circuits by connecting ac-circuit concepts to bioelectrical impedance analysis (BIA) using a custom-designed educational BIA device. The activity shows how a BIA device works and how current, voltage, and impedance measurements relate to bioelectrical characteristics of the human body. From this, useful observations can be made including body water, fat-free mass, and body fat percentage. The laboratory is engaging to pre-health and life-science students, as well as engineering students who are given the opportunity to observe electrical components and construction of a commonly used biomedical device. Electrical concepts investigated include alternating current, electrical potential, resistance, capacitance, impedance, frequency, phase shift, device design, and the use of such topics in biomedical analysis. @ 2014 American Association of Physics Teachers.
\end{abstract}

[http://dx.doi.org/10.1119/1.4866276]

\section{INTRODUCTION}

Drawing from the fields of physics, biology, medicine, physiology, and fitness sciences, we have developed a physics laboratory activity that introduces $R C$ electric circuits in conjunction with Bioelectric Impedance Analysis (BIA). Designed to demonstrate the electrical properties of the human body as relevant to medical science, the activity involves students in the dual analysis of both physical and biological systems. The concepts of impedance and frequency dependence are explained using $R C$ circuits and a cellularlevel analysis of body tissue. Experiments are performed on both of these systems using an educational BIA device we custom designed for this activity.

In the BIA laboratory described here, students acquaint themselves with the concepts of resistance, capacitance, impedance, and phase shifts in ac circuits. Circuits are constructed to emulate the bioelectrical behavior of the body, and an educational BIA device is used for measurements. Students are invited to make measurements on their own bodies using the same device. The data are compared with empirical fits for impedance and body composition, which students use to calculate their own body fat percentage and fat-free mass. These estimates are compared with measurements taken by a commercially available BIA device. Single- and multiple-frequency analyses are performed, exposing students to different circuit models of the body in an active exploration of ac circuitry. Finally, student attitudes were surveyed before and after the laboratory and the results are discussed in the final section of this paper.

\section{BACKGROUND}

\section{A. Motivation}

Although students in science courses have widely varying goals, a foundational knowledge of science and its practical application are necessary for those entering science, technology, engineering, and mathematics (STEM) disciplines. For life-science and pre-health students, traditional physics courses often do not meet the objectives of an adequate physical sciences background for their intended fields. ${ }^{1,2}$ We note that the new guidelines of the Medical College Admission Test (MCAT) stress interdisciplinary learning, and goals set forth by the American Association of Medical Colleges for future physicians include not only having a solid background in science but also to be prepared to use new advancements in science for ongoing professional development. ${ }^{3}$ Such goals are enhanced with better foundational understanding of the physics behind the wide array of medical technologies currently employed. A working knowledge base is needed for the continuous process of improving and applying technologies in biomedical engineering.

This laboratory exercise enhances undergraduate preparation for medical and all STEM fields by teaching physics through the application of technology, which in this instance is the use of electric circuit models for medical assessment. Algebra-based introductory physics courses can make use of this laboratory, although the subject matter is rich enough to challenge more advanced undergraduate physics or biomedical engineering majors. There has been an ongoing discussion concerning learning styles in students and their relevance to teaching methods. ${ }^{4}$ By giving students the opportunity to measure their own body's electrical impedance using BIA and participate in an active area of research, a wider positive student response to learning is anticipated. One may look to popular models of learning styles to understand the importance of multiple types of learning activities. For example, the VARK model of learning differentiates the needs of visual, aural, read/write, and kinesthetic learning styles. ${ }^{5}$ Although the visual and read/write styles should already be stimulated by the laboratory activity, kinesthetic learners will be more engaged with BIA measurements by their preference for activities requiring interacting with the environment. It has been suggested that fostering personally relevant activities in education results in increased learning overall. ${ }^{6}$ Scientific "story making" that emphasizes the learner's connection with a personal value system has been articulated in a new model for scientific learning. ${ }^{7}$ By directing a student's personal involvement in the laboratory exercises, our BIA laboratory works to create knowledge through connection. The BIA laboratory forms an extension from personal experiences of BIA at a gym, as part of a fitness regime, 
as health analysis, or with the student's opinions concerning these issues.

\section{B. Body composition}

BIA exists at the interposition of body composition analysis and bioelectrical analysis. The study of body composition is already very interdisciplinary, involving mathematics, physics, chemistry, biology, nutrition science, and other disciplines. ${ }^{8}$ Bioelectrical analysis surveys the electrical properties of tissue and can be used to analyze body composition models. While there are numerous methods to measure body composition, many either suffer from having low accuracies (caliper tests), being difficult to perform (densitometry), or are potentially harmful (dual-energy x-ray absorptiometry DXA). ${ }^{8}$ BIA avoids some of these drawbacks by taking advantage of the conductive properties of the human body.

One of the uses of BIA is to calculate the fat-free mass (FFM), or lean mass, of a person based on measurements of the electrical characteristics of the body and empirical data. There are two main electrical properties that characterize body tissues, resistance and capacitance. Cell membranes conducting an electrical current behave similar to capacitors. 9,10 Due to their ionic nature body fluids are good conductors, while fat cells are not. ${ }^{11}$ Bone is also considered a non-conductor under typical BIA conditions. ${ }^{12}$ The resistive measurement of BIA, therefore, relates only to soft tissue hydration.

Total body water (TBW) can be broken down into extracellular water (ECW) and intracellular water (ICW). Since cell membranes have a capacitive nature, body impedance is frequency dependent. Current flows primarily through ECW at low frequencies because cell membranes are essentially nonconductive. At higher frequencies, the reactance of cell membranes drops and current traverses ICW as well as ECW, as illustrated in Fig. 1(a). ${ }^{10}$ Once these components are obtained, lean tissue mass can be calculated because physiological constants relate the two. ${ }^{9}$ These correlations are based on large samplings of healthy individuals. ${ }^{13}$

\section{Single frequency BIA}

The most validated method of obtaining TBW is single frequency BIA (SF-BIA). ${ }^{9}$ At a single frequency, the body

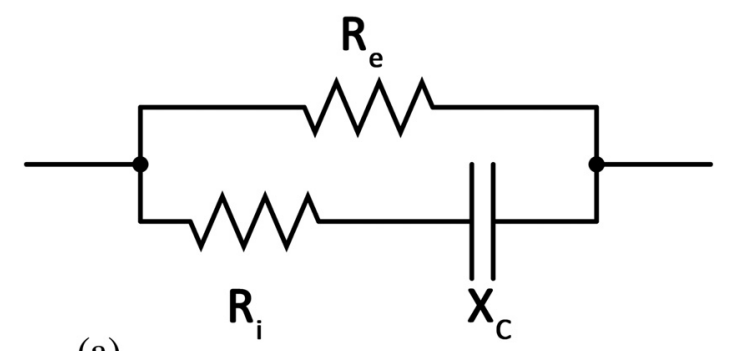

(a)

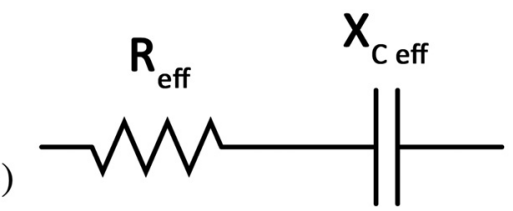

Fig. 1. Circuit models of the body: (a) $R_{\mathrm{e}}$ is the resistance of ECW, $R_{\mathrm{i}}$ is the resistance of ICW, and $X_{\mathrm{c}}$ is cellular membrane reactance; (b) For a single frequency, the effective resistance and reactance of the circuit in (a) can be found and the body can be modeled as a series $R C$ circuit. can be represented as a simple circuit with a resistor representing the effective resistance of TBW in series with a capacitor representing cell membranes, as shown in Fig. 1(b). Body impedance is typically measured at $50 \mathrm{kHz}$ and empirical relationships are used to derive fat-free mass (FFM) from the measured effective resistance. SF-BIA equations are empirical but are based on the relationship between the resistance $R$, resistivity $\rho$, and the dimensions of a cylindrical object as in

$$
R=\rho \frac{L}{A}=\rho \frac{L^{2}}{V},
$$

where $L, A$, and $V$ are the length, cross-sectional area, and volume of the cylinder, respectively. If one wants to relate the resistance to the mass one can substitute $V=m / d$, where $d$ is the density of the object and get

$$
m=\rho d \frac{L^{2}}{R} .
$$

As a first approximation one can consider an impedance measurement from hand-to-hand as having a cylindrical geometry. A person's height $H$ is approximately equal to the distance between fingertips with outstretched arms and may be substituted for $L$. The FFM follows a relationship similar to Eq. (2) but because of the complexity of the system, the density and resistivity of the body are replaced by an empirical constant $C$, so that

$$
\mathrm{FFM}=C \frac{H^{2}}{R} .
$$

A fully empirical relationship for FFM includes extra terms dependent on weight, age, gender, electrode placement, and other factors. An example of an empirical model for FFM will be given in the experimental section.

\section{Multi-frequency BIA and Bioelectrical Impedance Spectroscopy}

A second method used to analyze body composition is multi-frequency BIA (MF-BIA). This method models the body more accurately as a parallel $R C$ circuit with resistive $\mathrm{ECW}$ on one branch and resistive ICW and cell capacitance on the second branch [Fig. 1(a)]. Impedance measurements are made over a range of frequencies, the assumption being that ECW is measured at low frequencies when cell membranes block current, and total body water is measured at high frequencies when current passes through cell membranes, traversing both ICW and ECW.

A third method is known as Bioelectrical Impedance Spectroscopy (BIS), which makes use of the Cole model. ${ }^{9,14}$ In this approach, body impedance is also measured over a range of frequencies. For each frequency the series equivalent circuit for the circuit in Fig. 1(a) is found and the effective reactances and resistances are graphed using a ColeCole plot. Information from this graph is then used to calculate both ECW and ICW.

Although BIA is used in general for hydration assessment in athletes, nutritional analysis, and to characterize body fluid levels for ill patients such as those in renal dialysis, the method of BIA used depends on the application. ${ }^{13}$ For example, ECW may be better analyzed by MF-BIA than SF-BIA, 
while the estimation of TBW has been shown to be better with MF-BIA than BIS for patients with particular illnesses. ${ }^{9}$

\section{E. Current scientific understanding of BIA}

The methods of BIA are not without their shortcomings. The calibration of BIA is accomplished using a standard reference method such as Dual-energy X-ray Absorptiometry (DXA). Any errors associated with this method may be propagated into empirical BIA fits. ${ }^{8}$ Although the assumption is made that current will pass through ICW only at high frequencies, this is an idealization and is not entirely true. ${ }^{11}$ Furthermore, despite strong empirical correlations between body impedance and body composition parameters, it is not thoroughly understood exactly why BIA works as accurately as it does. ${ }^{10}$ Rather than a drawback, however, we see this as an opportunity for laboratory students. When confronted with new frontiers in science that test the limits of theory, there is the possibility to engage in open-ended inquiry. While exploring well-understood circuit components, students can extend these concepts and explore a real world problem without clean and simple answers, more closely resembling the experimental research of biomedical systems.

\section{DESIGN OF THE EDUCATIONAL BIA DEVICE}

BIA measurements are taken by injecting a small alternating current into the body. By comparing the potential across the body to one across a known reference resistor, it is possible to determine the equivalent electrical components that model the body. BIA devices for consumer use are frequently employed in fitness centers and integrated into some bathroom scales. Unlike most medical-grade equipment a consumer BIA device is inexpensive $;{ }^{15}$ however, its internal functions represent a black box with proprietary algorithms that output a person's body fat percentage (BF\%) or FFM. On the other hand, standard voltage probes and data collection systems used in undergraduate laboratories are not designed to measure the high frequencies used in BIA. The use of an oscilloscope would not only be difficult for many pre-health majors, it also would pose safety concerns when leads from a wall-powered scope are connected to the body. To eliminate this risk and to explore the mechanism of how ac current is used in BIA, we designed our own battery powered educational BIA device, which is shown in Fig. 2.

While a typical consumer device operates at a fixed frequency of $50 \mathrm{kHz}$, the educational device allows for a variable frequency $(20 \mathrm{~Hz}$ to $450 \mathrm{kHz})$ and the exploration of both BIS and MF-BIA. As part of the activity, students use an inexpensive frequency counter ${ }^{16}$ to measure the frequency settings of the device. A safety requirement of BIA devices is that the maximum current produced never exceeds $800 \mu \mathrm{A} .{ }^{13}$ The educational BIA device outputs a constant current amplitude of $100 \mu \mathrm{A}$. The total cost to build the described educational BIA device is less than \$75. Reference 17 provides full specifications and circuit diagrams of the device.

The BIA device is based on the AD8302 Gain and Phase Detector (Fig. 3), which can be used to find the impedance and phase of an $R C$ circuit $^{18}$ and has already been shown to work in a BIA device. ${ }^{19}$ The Gain and Phase Detector takes two sinusoidal signals as inputs and outputs two de potentials, $V_{\text {phase }}$ and $V_{\text {mag. }}$. Here $V_{\text {phase }}$ is proportional to the phase shift $\Delta \varphi$ between the inputs, and $V_{\text {mag }}$ is proportional to the

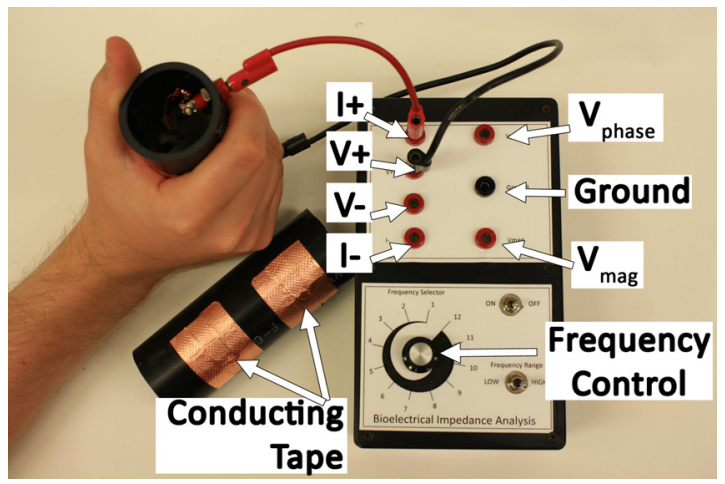

Fig. 2. The custom built, educational BIA device and hand-held electrodes used in the laboratory activity. The electrodes are made from conducting tape, banana jacks, and plastic tubing. During a measurement students hold the two electrodes, which are connected with long leads to the device, with their arms outstretched at chest level. A battery-powered voltmeter is connected to ground and either $V_{\text {phase }}$ or $V_{\text {mag }}$. (See Fig. 3 for the block diagram of the device.)

logarithm of the ratio of the connected impedance $Z$ to the reference resistance $R_{\text {ref }}$. Thus, we have $V_{\text {phase }} \propto \Delta \varphi$ and $V_{\mathrm{mag}} \propto \log _{10}\left(Z / R_{\text {ref }}\right)$. (See Ref. 18 for the full conversion formulas.)

There are multiple methods of electrode placement in BIA including hand-to-hand, foot-to-foot, and hand-to-foot. ${ }^{13}$ Our device uses hand-to-hand electrodes that are designed with conductive tape on plastic tubing (Fig. 2). Four electrodes are needed for BIA, two electrodes to carry current and two to measure the potential across the body. Tetrapolar voltage probes minimize the effect of the skin-electrode impedance $Z_{\text {contact }}$ on the measurement of the body's impedance $Z_{\text {body }}$ (Fig. 4). ${ }^{20}$

\section{LABORATORY EXERCISES}

The major goals of the laboratory exercises are to develop a foundational understanding of how resistors and capacitors

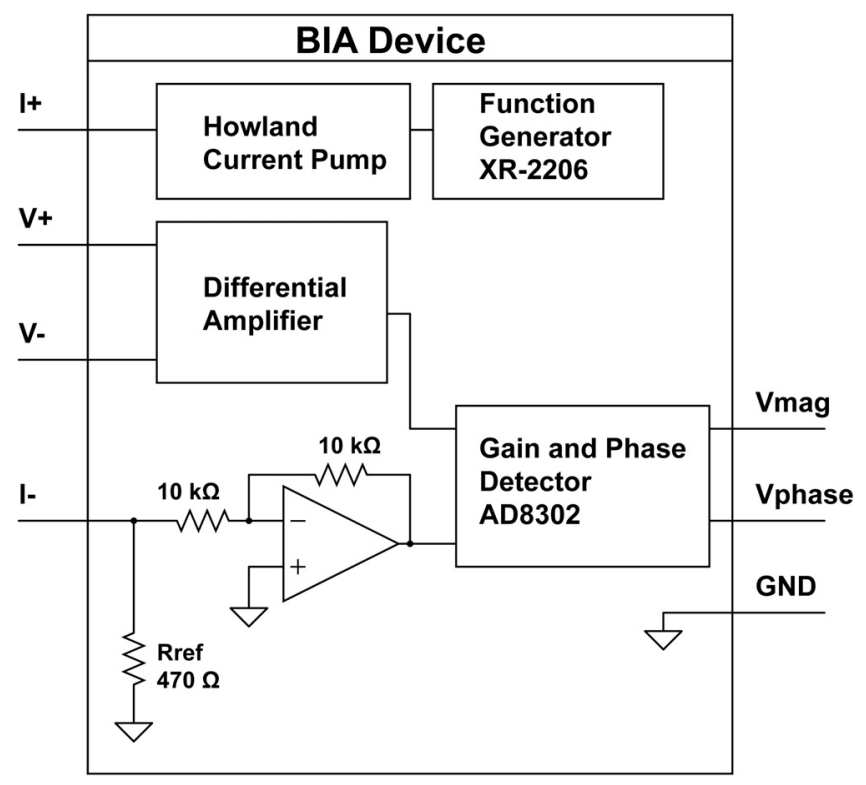

Fig. 3. Block diagram of the educational BIA device designed and constructed for this laboratory. The inverting amplifier acts as a buffer to the input of the Gain and Phase Detector. (See Ref. 17 for more information.) 


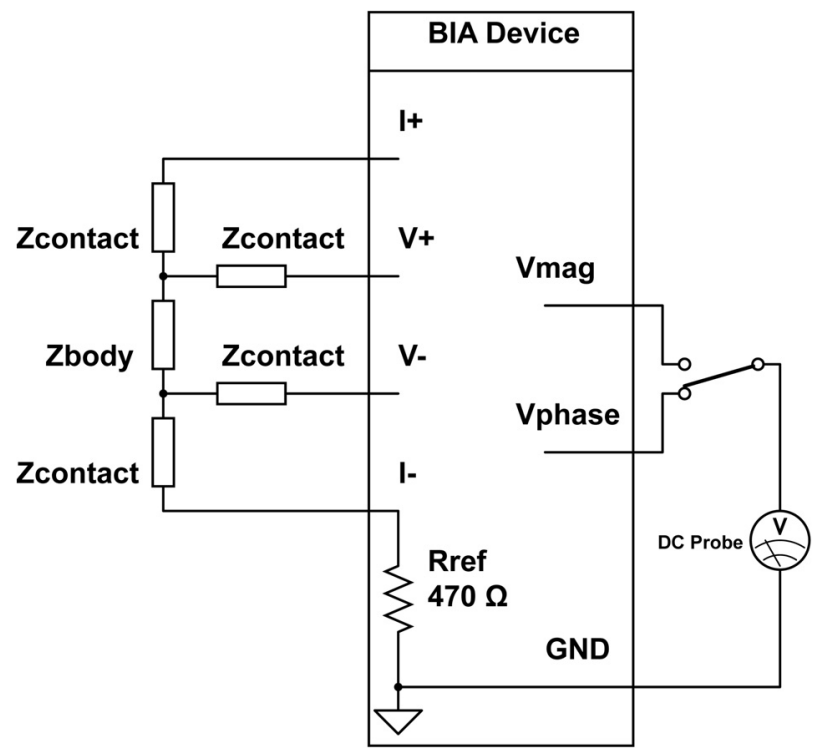

Fig. 4. Circuit representation of the human body connected to the educational BIA device; $Z_{\text {body }}$ represents the impedance of the body and $Z_{\text {contact }}$ is the impedance at the skin-electrode interface.

behave in ac circuits, to demonstrate how the body can be modeled as an $R C$ circuit, and to show how the electrical characteristics of the body are used to elicit physiological information. Specifically, students explore the frequency dependence of resistance, reactance, impedance, and phase angle in $R C$ circuits using tools like phasor diagrams or, for more advanced students, complex analysis of impedances. These concepts are introduced and demonstrated with a series $R C$ circuit. The equations expressing impedance and phase angle for a series $R C$ circuit with a resistor and capacitor are given by $Z=\sqrt{R^{2}+X_{C}^{2}}$ and $\tan \varphi=X_{c} / R$, where $Z$ is impedance, $R$ is resistance, $X_{C}$ is capacitive reactance, and $\varphi$ is phase angle. These equations can be solved for $R$ and $X_{C}$, resulting in $R=\sqrt{Z^{2} /\left(1+\tan ^{2} \varphi\right)}$ and $X_{C}=R \tan \varphi$.

\section{A. BIA for a simple $R C$ circuit}

During the first part of the laboratory, experimentation with a series $R C$ circuit is undertaken to confirm the frequency dependence of phase angle and impedance. This also serves as an introduction to the functionality of the educational BIA device. Students analyze the potentials across circuit components when a resistor and capacitor are connected in series to the educational BIA device (Fig. 5). At low frequencies $(<5 \mathrm{kHz})$ students observe the alternating signals directly and in real time using Vernier differential voltage probes $^{21}$ and LoggerPro (Fig. 6). Alternatively, one can use equipment from other manufactures, such as PASCO, or an oscilloscope, which has the disadvantage of additional complexity for students. At high frequencies $(>5 \mathrm{kHz})$ students measure the voltage ratios and phase differences from the dc outputs of the educational BIA device [Fig. 5(b)] as described above by $V_{\text {phase }}$ and $V_{\text {mag }}$.

\section{Impedance and phase shift from the time resolved ac signal at low frequencies}

To take measurements in the low-frequency range (20-500 $\mathrm{Hz}$ ), differential voltage probes are placed across the series circuit and the reference resistor internal to the educational BIA device, as shown in Fig. 5(a). The best curve fits of the potentials are used to measure the phase difference and gain of the two signals (Fig. 6). Since the resistors and capacitor are in series, all three components carry the same current and the impedance and phase shift can be calculated according to $Z=R_{\text {ref }} V_{Z} / V_{\text {ref }}$ and $\Delta \varphi=\left|\varphi_{\text {ref }}-\varphi_{z}\right|$, where theoretical values for $Z$ and $\Delta \varphi$ are calculated according to their definitions given above.

\section{Impedance and phase shift from dc output at high frequencies}

Above $5 \mathrm{kHz}$, the educational BIA device is used to obtain values for impedance and phase as described in Sec. III, connecting the BIA device as shown in Fig. 5(b). Students plot
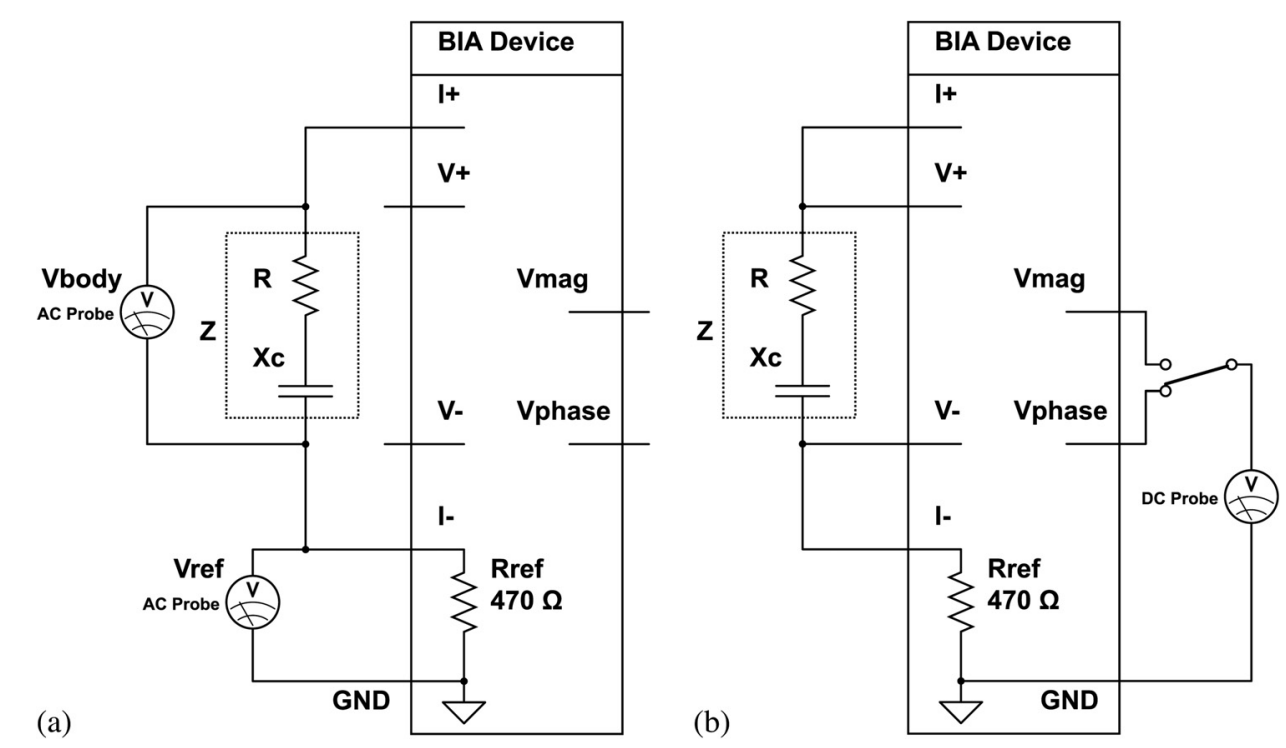

Fig. 5. Series $R C$ circuit connected to the educational BIA device. The dashed box is the total impedance $Z$ of the $R C$ series circuit. (a) Use of external differential voltage probes to measure the time-resolved potentials $V_{\text {body }}$ and $V_{\text {ref }}$ at low frequencies; (b) Use of a dc voltage probe to measure impedance and phase at high frequencies. 


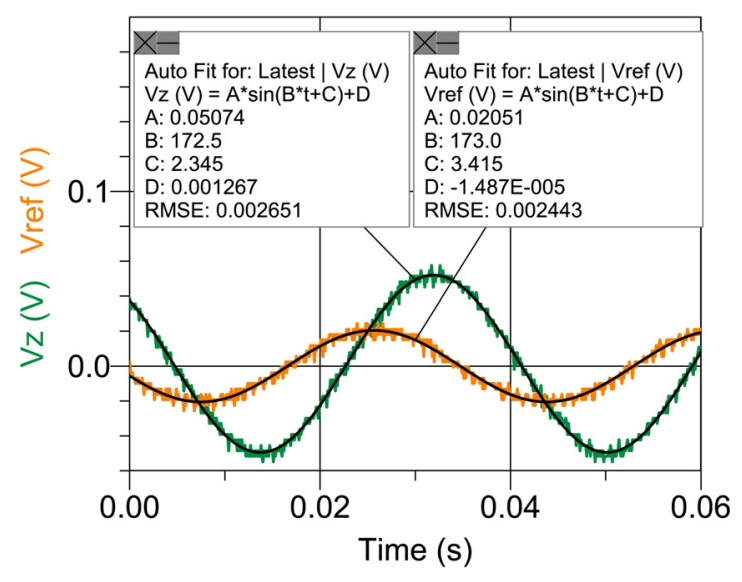

Fig. 6. Plot of the potentials across the series $R C$ circuit $\left(V_{Z}\right)$ and the reference resistor $\left(V_{\text {ref }}\right)$. The amplitudes $(\mathrm{A})$ and phases $(\mathrm{C})$ from the fits are used to calculate $Z$ and $\Delta \varphi$ for the $R C$ circuit according to the equations in Sec. IV. For the signals shown, $Z=1160 \Omega$ and $\Delta \varphi=61^{\circ}$. Note that due to the low current amplitude of the device, the potentials have small magnitudes and digitization errors are visible. The components of the circuit are $R=470 \Omega$ and $C=6 \mu \mathrm{F}$.

the experimental and theoretical values for impedance vs frequency and phase shift vs frequency (Fig. 7). One can observe the characteristic inverse relationship of the impedance vs frequency and the leveling off of the resistance of the circuit for high frequencies. Students can also observe how the phase shift decreases with increasing frequency. This part of the laboratory explores many of the key concepts of ac circuits taught in introductory physics and helps students get familiar with the educational BIA device.
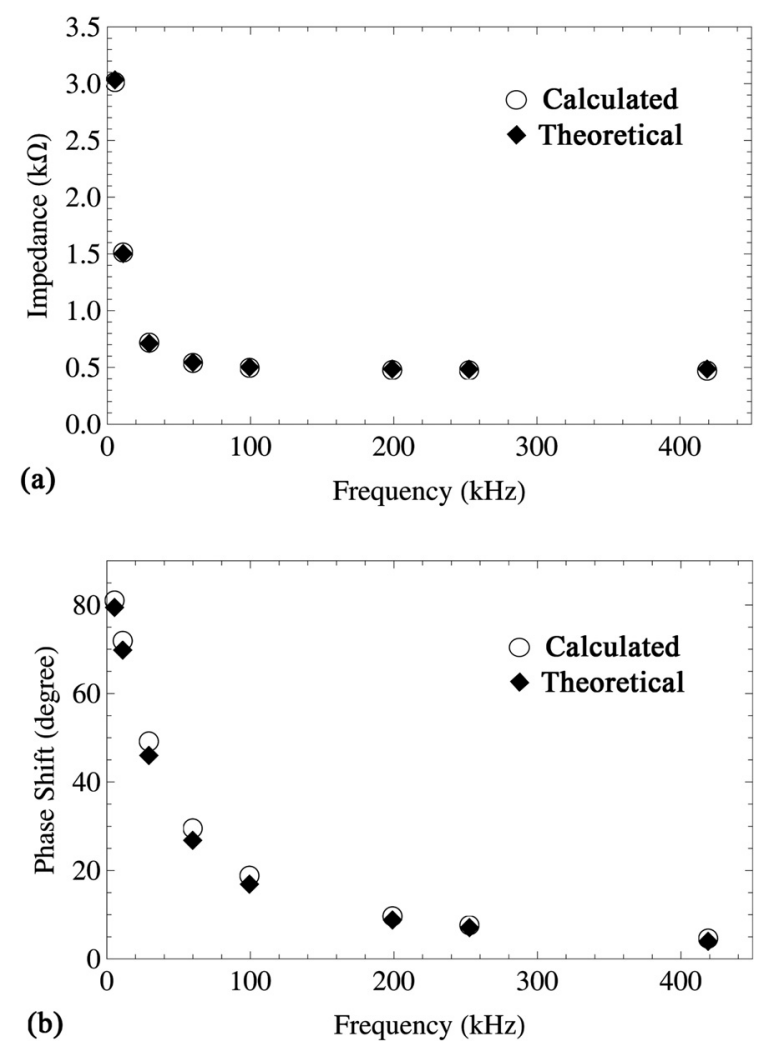

Fig. 7. Measurements of a series $R C$ circuit using the educational BIA device $(R=470 \Omega$ and $C=10 \mathrm{nF}$ ): (a) Impedance $Z$ vs frequency; (b) Phase shift $\Delta \varphi$ vs frequency.

\section{B. Single frequency BIA of the human body}

\section{Empirical calibration of the educational BIA device}

In the next section of the laboratory, bioelectrical impedance analysis of the human body is introduced. Equation (1) is presented to students and the dependence of resistance on length, or in this case height of the body, is discussed. If the instructor has time, this concept can be explored with students by measuring the resistance of cylinders of different geometries and resistivities. As stated in the BIA background section, Eq. (3) is only suitable as a first approximation for FFM. Published studies of BIA use fitting factors to improve the correlation between impedance and FFM. Fitting factors are found by plotting BIA measurements against a "gold standard" for body composition analysis, such as DXA. ${ }^{13}$

To find appropriate fitting factors for our educational BIA device, we surveyed 32 volunteers who provided their height, age, and gender. The participants' weights were measured using a force plate. Impedance and phase information was obtained using the BIA device, and $R$ was found using the equations in Sec. IV. An estimate for FFM for each participant was found using the collected data and a published BIA equation for FFM chosen based on its high correlation coefficient. ${ }^{22}$ Because our device measures hand-tohand impedance, it was not sufficient to use the published equation, which was generated based on hand-to-foot impedance measurements. Therefore, the participant's FFM was also measured with a commercially manufactured hand-tohand BIA device, the OMRON HBF-306C, ${ }^{15}$ which acted as our "gold standard." The equation that is programmed into the OMRON to calculate FFM is proprietary and therefore unavailable. $^{23}$ The measurements from the OMRON were plotted versus the published equation, and the slope and the offset from the linear fit of this plot were used to modify the published equation to yield a new empirical equation for FFM based on the impedance and phase measurements from the educational BIA device. The resulting equation is

$$
\begin{aligned}
\text { FFM }= & 0.360 \cdot \frac{\text { Height }^{2}}{R}+0.162 \cdot \text { Height }+0.289 \\
& \cdot \text { Weight }-0.134 \cdot \text { Age }+4.83 \cdot \text { Gender }-6.83,
\end{aligned}
$$

where FFM is measured in $\mathrm{kg}$, height in $\mathrm{cm}$, weight in $\mathrm{kg}$, age in years, and gender is either 1 for men or 0 for women. This modified FFM equations had good agreement with the OMRON device, as shown in Fig. 8. Published BIA equations commonly do not report the units of the coefficients, ${ }^{13}$ though it may be a useful exercise for students to determine what they should be. As with any empirical relationship, the equation may be modified and improved upon as more people are included in the calibration. Students are encouraged to explore how the individual factors affect the correlation and it may be an interesting student project to explore other empirical relationships for the given input parameters.

\section{Measuring body fat percentage and fat-free mass}

Students use the empirical fit in Eq. (4) to measure the body impedance of a volunteer. One electrode pair (Fig. 2) is held in each hand so that a complete circuit is created across the body. It is important to note that the electrodes must be held with arms outstretched and held at chest level, to obtain 


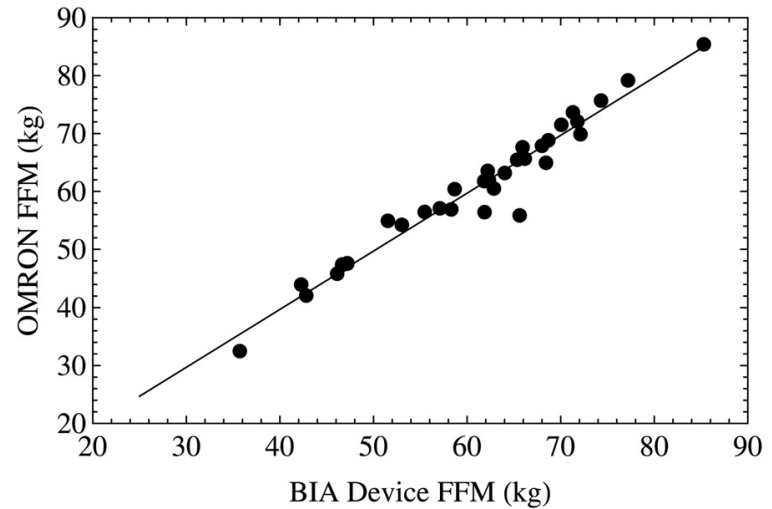

Fig. 8. Correlation of FFM as measured by the OMRON and by the educational BIA device. Twenty-three men and nine women were tested.

consistent results. ${ }^{15}$ Students measure the volunteer's $V_{\text {phase }}$ and $V_{\text {mag }}$ at a frequency of $50 \mathrm{kHz}$ and calculate $Z$ and $\Delta \varphi$ using the equations in Sec. IV, as they did for the $R C$ circuit. From $Z$ and $\Delta \varphi$, the effective $R$ and $X_{C}$ are then calculated for the single-frequency series-equivalent circuit of Fig. 1(b). The student enters the value for $R$ obtained with the custom BIA device, along with the height, weight, age, and gender into Eq. (4). Figure 9 shows the sequence of these calculations. (If time is a constraint, these calculations can be pre-coded into a spreadsheet.) The OMRON BIA device (which also operates at $50 \mathrm{kHz}$ ) is then used to measure $\mathrm{BF} \%$ for the volunteering student, and the two values are compared (Table I). The BIA device returns a value for FFM, but the OMRON returns a person's BF\%. Either measurement can be converted using the individual's weight. It should be noted that the empirical models assume normal body hydration levels and that the measurement is taken several hours after eating. Because students are not asked to follow the strict regimen required for highly accurate FFM measurements, some variation in the results from a person's true FFM are possible. ${ }^{15}$

Alternatively, instructors can also use known components to model the human body including skin-electrode impedances $Z_{\text {contact }}$ as shown in Fig. 4. This circuit allows for a quantitative and more in-depth analysis of the tetrapolar electrode setup.

\section{Multi-frequency BIA and bioelectrical impedance spectroscopy}

A BIA measurement as taken by a consumer device such as the OMRON is performed at a single frequency. Multifrequency BIA is first examined using a more accurate circuit representation of the body as shown in the parallel circuit of Fig. 1(a). Students measure $Z$ and $\Delta \varphi$ over a range of frequencies and calculate the effective resistance and reactance of the series equivalent circuit using Sec. IV equations. These values are graphed with reactance on the $y$-axis and resistance on the $x$-axis; such a graph is often referred to as a Cole-Cole plot. The result is a semi-circular curve whose center is depressed below the $x$-axis (Fig. 10). The resistances $R_{0}$ and $R_{\infty}$, the low- and high-frequency extremes, are the intersections of the curve with the $x$-axis. In BIS, these resistances are used to calculate ECW and ICW. ${ }^{13}$ The peak of the semicircle for an average human body occurs near $50 \mathrm{kHz} .{ }^{10}$ At this frequency, current passes through both ICW and ECW. Since the measured values for these variables will be compared against the theoretical values, $R_{\text {eff }}$ and $X_{C \text {,eff }}$ have to be found for each frequency. Complex analysis can be used with upper-level physics or biomedical engineering students to derive these expressions or the results can be stated and used by introductory students. The effective resistance and reactance are given by

$$
R_{\mathrm{eff}}=\frac{R_{e} R_{i}\left(R_{e}+R_{i}\right)+R_{e} X_{i}^{2}}{\left(R_{e}+R_{i}\right)^{2}+X_{i}^{2}}
$$

and

$$
X_{C, \mathrm{eff}}=\frac{R_{e}^{2} X_{i}}{\left(R_{e}+R_{i}\right)^{2}+X_{i}^{2}} .
$$

Whether or not the students perform the complex analysis necessary to derive these equations, they can analyze the salient features of the graph. Figure 10(a) shows the Cole-Cole plot for an $R C$ parallel circuit such as shown in Fig. 1(a). Students will find that at low frequencies, the capacitor acts as a break in the circuit and the effective resistance essentially becomes $R_{0}=R_{e f f}=R_{e}$. At high frequencies, the capacitor acts as a short and the circuit becomes a parallel resistive circuit, described by the equation

$$
R_{\infty}=R_{\mathrm{eff}}=\left(\frac{1}{R_{e}}+\frac{1}{R_{i}}\right)^{-1}
$$

Next, a student volunteer repeats the set of MF-BIA measurements with the hand-held electrodes over a range of frequencies. Using the measured resistances and reactances of the human body, a new Cole-Cole plot is constructed, as shown in Fig. 10(b). Students compare the salient features of this plot with that of the parallel circuit. The peak of the semi-circular arc for the human body is near $50 \mathrm{kHz}$, thus indicating to students why SF-BIA uses that frequency. Students also find the resistances $R_{0}$ and $R_{\infty}$ for their own bodies based on their Cole-Cole plot and calculate $R_{e}$ and $R_{i}$ for the parallel-circuit model [Fig. 1(a)] using $R_{0}=R_{\text {eff }}=$ $R_{e}$ and Eq. (7).

\section{Student responses}

Before we implemented the lab for the first time, we conducted an anonymous survey of 292 general physics students

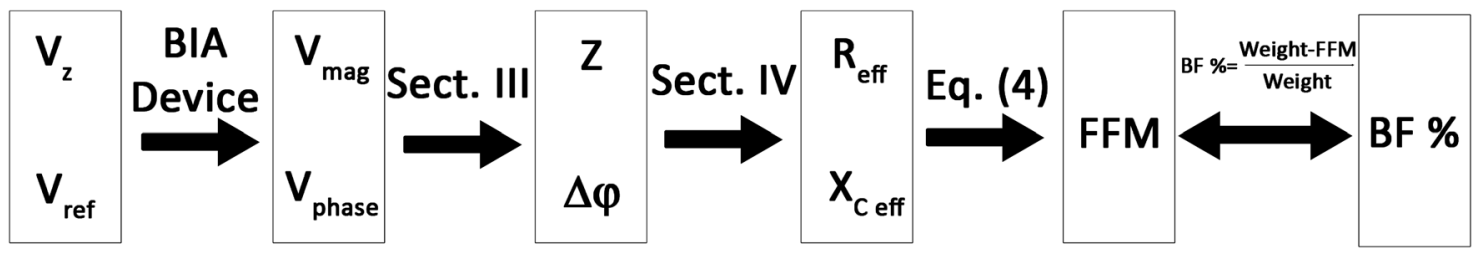

Fig. 9. Summary of the measurements and calculations used to find the FFM in Table I. 
Table I. Comparison of FFM and BF\% measurements by the custom BIA device and the OMRON. Coincidentally, both students had the same BF\% despite having different input parameters.

\begin{tabular}{|c|c|c|c|c|c|c|c|c|c|c|c|}
\hline \multirow[b]{2}{*}{ Student } & \multirow[b]{2}{*}{$f(\mathrm{kHz})$} & \multirow[b]{2}{*}{$V_{\text {phase }}(\mathrm{V})$} & \multirow[b]{2}{*}{$V_{\text {mag }}(\mathrm{V})$} & \multirow[b]{2}{*}{$Z(\Omega)$} & \multirow[b]{2}{*}{$\Delta \phi($ degree $)$} & \multirow[b]{2}{*}{$R_{\text {eff }}(\Omega)$} & \multirow[b]{2}{*}{$X_{\mathrm{C} \text { eff }}(\Omega)$} & \multicolumn{2}{|c|}{ BIA Device } & \multicolumn{2}{|c|}{ OMRON } \\
\hline & & & & & & & & FFM $(\mathrm{kg})$ & $\mathrm{BF} \%$ & FFM (kg) & $\mathrm{BF} \%$ \\
\hline $\mathrm{A}^{\mathrm{a}}$ & 50.4 & 1.836 & 1.040 & 685.5 & 4.7 & 683.2 & 56.5 & 62.8 & 24.7 & 63.8 & 23.5 \\
\hline $\mathrm{B}^{\mathrm{b}}$ & 50.4 & 1.857 & 1.081 & 800.3 & 2.8 & 799.3 & 39.3 & 46.1 & 24.7 & 46.8 & 23.6 \\
\hline
\end{tabular}

${ }^{\mathrm{a}}$ Height $=175.3 \mathrm{~cm}$, Weight $=83.5 \mathrm{~kg}$, Age $=29$ yrs., and Gender $=1$ (male).

${ }^{\mathrm{b}}$ Height $=167.6 \mathrm{~cm}$, Weight $=61.2 \mathrm{~kg}$, Age $=34$ yrs., and Gender $=0$ (female).

at Portland State University and found that 58.6\% said they would volunteer for the body fat percentage measurement, $17.1 \%$ said they would volunteer if nobody else in the group would, and $22.9 \%$ said they would not want to volunteer ( $1.4 \%$ did not respond to the survey). The percentages were similar when broken up into algebra-based and calculusbased physics students and also when separated into female and male students.

The BIA laboratory was conducted first at PSU in a biomedical physics course (see Refs. 2 and 24 for course details). The students consisted of 21 pre-health and biomedical-physics students working in groups of two to four, and the instruction took place over two 130-min class periods. During the first session, the instructor spent one hour discussing the various methods to measure a person's body composition. This was followed by a half-hour review of ac circuits and a half-hour theoretical introduction of the BIA device. Most students had seen ac circuits in a previous general physics course. The following class period was spent on the laboratory activity, and we provided a spreadsheet to students that had many of the longer equations pre-coded. It was emphasized that the body fat percentage measurements

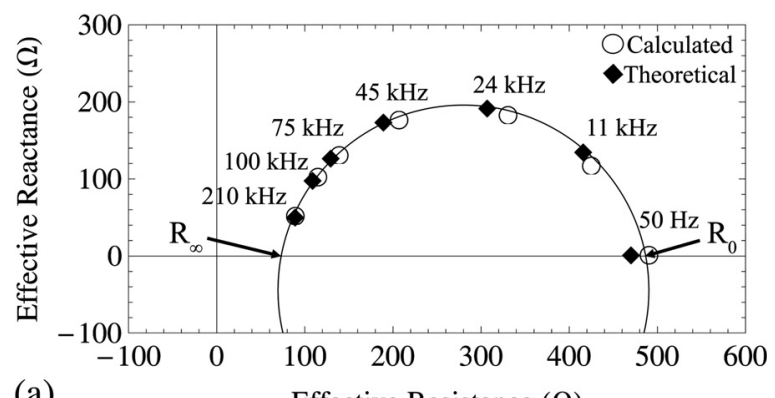

(a)

Effective Resistance $(\Omega)$

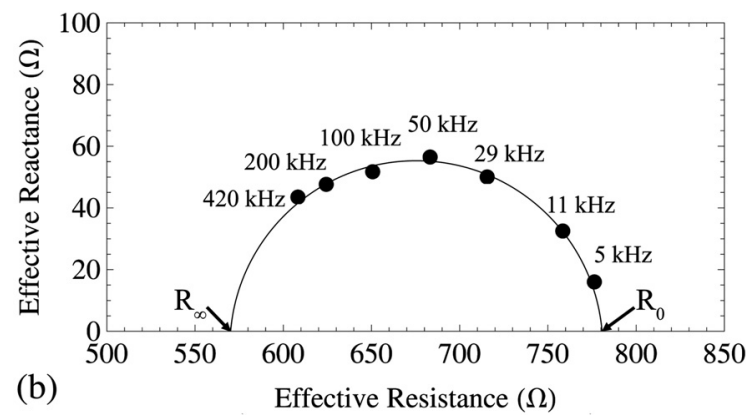

Fig. 10. BIS measurements by the educational BIA device. (a) Cole-Cole plot of a parallel circuit as in Fig. 1(a) using $R_{e}=470 \Omega, R_{i}=100 \Omega$, and $C=10 \mathrm{nF}$. Differential voltage probes were used for the $50-\mathrm{Hz}$ data point. The measured $R_{0}$ and $R_{\infty}$ are in good agreement with $R_{0}=R_{\text {eff }}=R_{e}$ and Eq. (7). (b) Cole-Cole plot of body impedance. Note that at the peak of the curve $f=50 \mathrm{kHz}$, which is the frequency normally used by SF-BIA. were voluntary. We found that the group dynamics were very positive. At least one student from every group volunteered for the measurement and many groups had multiple students take their own measurements even though the provided spreadsheet had space for only one measurement per group.

At the end of the term, a survey was completed by 18 of 21 students. The results showed that 15 students checked "I was interested in volunteering for this part and felt comfortable with it," 2 checked "I volunteered for this part because no one else in my lab group would do it," and one checked "I did not feel comfortable volunteering for this part." Nine of 18 students had used a BIA device before to measure their body fat percentage.

We recognize that sensitivity is required on the part of the instructor to make sure body consciousness is respected and generating an environment where students feel comfortable is of the utmost importance. The response from our students was very positive and it appears that they found the personal involvement very engaging.

\section{CONCLUSION}

Deconstructing BIA in an undergraduate physics laboratory gives students both conceptual and practical exposure to the importance of physics in medicine beyond a traditional physics laboratory. Conceptually, students are exposed to principles of $R C$ circuits by connecting medical inquiry with the function of a common device. Students practice circuit analysis by relating body impedance to simple electrical components. Outcomes range from an increased understanding of concepts needed for a solid physics background for STEM majors to an integrated, real-world appreciation for the necessity of that understanding. In addition, $R C$ circuits with ac signals, resistance, capacitance, impedance, frequency, electrical properties of biological tissue, and device construction are explained and practiced to varying degrees. The complexity of the BIA laboratory is at an introductory physics level but can be expanded to challenge those in upper division courses. This laboratory activity allows students to relate in a personal manner to an otherwise abstract concept.

${ }^{\text {a)} E l e c t r o n i c ~ m a i l: ~ e m y l o t t @ p d x . e d u ~}$

${ }^{1}$ D. C. Meredith and E. F. Redish, "Reinventing physics for life-sciences majors," Phys. Today 66(7), 38-43 (2013).

${ }^{2}$ G. R. VanNess and R. Widenhorn, "Engaging the community through an undergraduate biomedical physics course," Am. J. Phys. 80, 1094-1098 (2012).

3 AAMC-HHMI Committee, "Scientific Foundations for Future Physicians," Washington, DC: Association of American Medical Colleges (2009). Manuscript retrieved from <http://www.hhmi.org/grants/pdf/08-209_ AAMC-HHMI_report.pdf/>. 
${ }^{4}$ N. Othman and M. H. Amiruddin, "Different perspectives of learning styles from VARK Model,” Proc. Soc. Behav. Sci. 7, 652-660 (2010).

${ }^{5}$ W. A. Drago and R. J. Wagner, "VARK preferred learning styles and online education," Man. Res. News. 27, 1-13 (2004).

${ }^{6} \mathrm{~K}$. J. Pugh and M. Girod, "Science, art, and experience: Constructing a science pedagogy from Dewey's aesthetics," J. Sci. Teach. Ed. 18, 9-27 (2007).

${ }^{7}$ R. Reilly and B. Kort, "The Science Behind the Art of Teaching Science: Emotional State and Learning," in Society for Information Technology \& Teacher Education International Conference, pp. 3021-3026 (2004).

${ }^{8}$ M. Siervo and S. A. Jebb, "Body composition assessment: Theory into practice,” IEEE Eng. Med. Bio. 29, 48-59 (2010).

${ }^{9}$ S. Grimnes and O. G. Martinsen, Bioimpedance and Bioelectricity Basics, 2nd ed. (Oxford, UK: Elsevier Ltd., 2008).

${ }^{10}$ K. R. Foster and H. C. Lukaski, "Whole-body impedance-what does it measure?,” Am. J. Clin. Nut. 64, 388S-396S (1996).

${ }^{11}$ A. Piccoli, P. Giordano, M. Guizzo, M. Rebeschini, A. Naso, and C. Cascone, "Equivalence of information from single versus multiple frequency bioimpedance vector analysis in hemodialysis," Kid. Int. 67, 301-313 (2005).

${ }^{12}$ A. Piccoli, L. Pillon, and F. Dumler, "Impedance Vector Distribution by Sex, Race, Body Mass Index, and Age in the United States: Standard Reference Intervals as Bivariate Z Scores," Nutrition 18, 153-167 (2002).

${ }^{13}$ U. G. Kyle, I. Bosaeus, A. D. De Lorenzo, P. Deurenberg, M. Elia, J. M. Gomez, B. L. Heitmann, L. Kent-Smith, J. C. Melchior, M. Pirlich, H. Scharfetter, A. M. W. J. Schols, and C. Pichard, "Bioelectrical impedance analysis-part I: Review of principles and methods," Clin. Nut. 23, 1226-1243 (2004).

${ }^{14}$ K. S. Cole and R. H. Cole, "Dispersion and absorption in dielectrics," J. Chem. Phys. 9, 341-351 (1941).

${ }^{15}$ OMRON HBF-306C Fat Loss Monitor Manual, OMRON Healthcare, INC., 1200 Lakeside Drive, Bannockburn, IL 60015, USA. Instruction manual available online at $<\mathrm{http} / /$ www.omronhealthcare.com/wp-content/uploads/ hbf-306c-instruction-manual.pdf/ $>$.

${ }^{16}$ VC3165 Intelligence Frequency Counter, Delli Industry (Hong Kong) Co., Limited, Shenzhen, China.

${ }^{17}$ See supplementary material at http://dx.doi.org/10.1119/1.4866276 for complete specifications and circuit diagrams for the Educational BIA Device.

${ }^{18}$ AD8302 Data Sheet 2002 Analog Devices, One Technology Way, PO Box 9106, Norwood, MA 02062-9106, USA.

${ }^{19}$ Y. Yang, J. Wang, G. Wu, F. Niu, and P. He, "Design and preliminary evaluation of a portable device for the measurement of bioimpedance spectroscopy," Physiol. Meas. 27, 1293-1310 (2006).

${ }^{20}$ G. Medrano, R. Bausch, A. H. Ismail, A. Cordes, R. Pikkemaat, and S. Leonhardt, "Influence of ambient temperature on whole body and segmental bioimpedance spectroscopy measurements," J. Phys.: Conf. Ser. 224, 012128-1-4 (2010).

${ }^{21}$ Differential Voltage Probe, Vernier Software \& Technology, 13979 S.W. Millikan Way, Beaverton, OR 97005, USA. Instruction manual available online at $<$ http://www.vernier.com/files/manuals/dvp-bta.pdf $>$.

${ }^{22}$ P. Durenberg, K. van der Kooy, R. Leenan, J. A. Westrate, and J. C. Seidell, "Sex and age specific prediction formulas for estimating body composition from bioelectrical impedance: A cross-validation study," Int. J. Obesity 15, 17-25 (1991).

${ }^{23}$ A. L. Gibson, V. H. Heyward, and C. M. Mermier, "Predictive accuracy of OMRON body logic analyzer in estimating relative body fat of adults," Intl. J. Sp. Nut. Exer. Met. 10, 216-227 (2000).

${ }^{24}$ W. Christensen, J. K. Johnson, G. R. Van Ness, E. Mylott, J. C. Dunlap, E. A. Anderson, and R. Widenhorn, "Developing and assessing curriculum on the physics of medical instruments," CBE-Life Sci. Ed., 12, 250-261 (2013).

\section{ALL BACK ISSUES ARE AVAILABLE ONLINE}

The contents of the American Journal of Physics are available online. AJP subscribers can search and view full text of AJP issues from the first issue published in 1933 to the present. Browsing abstracts and tables of contents of online issues and the searching of titles, abstracts, etc. is unrestricted. For access to the online version of AJP, please visit http://aapt.org/ajp.

Institutional and library ("nonmember") subscibers have access via IP addresses to the full text of articles that are online; to activate access, these subscribers should contact AIP, Circulation \& Fulfillment Division, 800-344-6902; outside North American 516-576-2270 or subs@aip.org.

APPT (individual) members also have access to the American Journal of Physics Online. Not a member yet? Join today http://www.aapt.org/membership/joining.cfm. Sign up for your free Table of Contents Alerts at http://www.ajp.aapt.org/features/toc_email_alerts. 